\title{
A generalized relationship for swirl decay in laminar pipe flow
}

\author{
T F AYINDE \\ Mechanical Engineering Department, King Fahd University of Petroleum and \\ Minerals, Dhahran 31261, Saudi Arabia \\ e-mail: ayinde@kfupm.edu.sa
}

MS received 24 July 2008; revised 28 January 2010; accepted 1 February 2010

\begin{abstract}
Swirling flow is of great importance in heat and mass transfer enhancements and in flow measurements. In this study, laminar swirling flow in a straight pipe was considered. Steady three-dimensional axisymmetric Navier-Stokes equations were solved numerically using a control volume approach. The swirl number distribution along the pipe length was computed. It was found that the swirl number at any location along the pipe length depends on the swirl number at inlet, the flow Reynolds number, the distance from the pipe inlet, the pipe diameter and the nature of the inlet swirl. A generalized relationship for swirl decay as a function of these parameters was then obtained by curve-fitting technique.
\end{abstract}

Keywords. Laminar pipe flow; axisymmetric; swirl number; forced vortex; free vortex.

\section{Introduction}

The concept of swirling flow in a pipe is important because there are numerous applications in which swirl is either desired or it is a nuisance. The use of swirl flow has been recognized as one of the most promising techniques for heat transfer augmentation (Chang \& Dhir 1995, Bali 1998, Li \& Tomita 1994) as well as mass transfer enhancement (Yapici et al 1994). On the other hand, swirl is credited with causing significant errors in flow measurements employing orifice plates, nozzles, and venturi tubes (Reader-Harris 1994, Parchen \& Steenbergen 1998).

Chang \& Dhir (1995) experimentally investigated heat transfer enhancement resulting from introduction of swirl. Their results showed that the heat transfer coefficient increased with swirl intensity. This was attributed to the high magnitude of maximum axial velocity near the wall (which produced high rate of heat flux from the wall) and high turbulence level in the middle region (which improved flow mixing). Similarly, Bali (1998) reported heat transfer enhancement, as well as higher pressure drop, in the flow when swirl was introduced. Li \& Tomita (1994) experimentally obtained correlations, in terms of swirl level, for static, dynamic and wall pressures.

In spite of the significance of swirling pipe flow in some industrial processes, there are no clear generalized methods in the literature to compute the decay of swirl. While the authors 


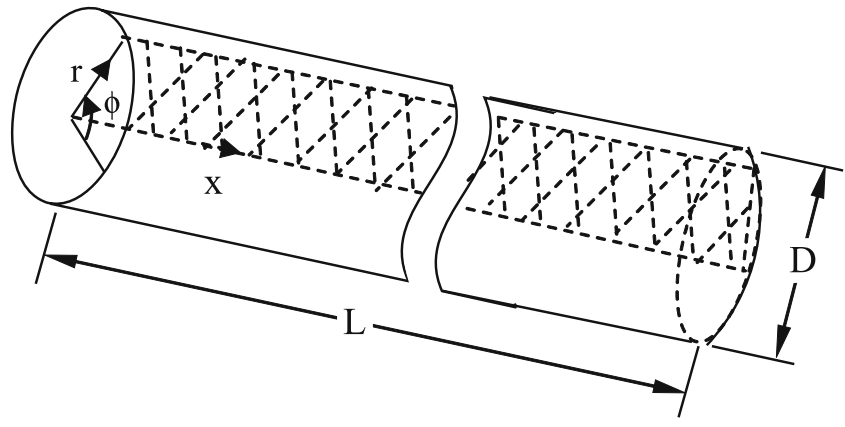

Figure 1. A schematic view of the pipe showing the coordinate system and the computational domain (hatched plane).

Li \& Tomita (1994); Parchen \& Steenbergen (1998) correlated the obtained swirl decay as a function of axial position normalized by pipe diameter only, Reader-Harris (1994) argued that swirl was also a function of the pipe friction factor. In order to obtain a generalized relationship for swirl decay in laminar pipe flow, numerical computations are performed for four different tangential velocity distributions at pipe inlet, four different levels of inlet swirl numbers and a wide range of Reynolds number (in the laminar regime). Such a generalized formula can be used as input in the existing correlations for heat transfer enhancement in pipe flow (Chang \& Dhir 1995, Bali 1998, Li \& Tomita 1994). It can also serve as a predictive tool for determining the downstream location where the swirl would have reduced to such an acceptable level that flow measurement can be performed with standard flow metering devices.

\section{Mathematical modelling}

\subsection{Flow domain}

The steady laminar incompressible and axisymmetric flow in a straight constant-diameter pipe is considered. The schematic view of the computational domain is shown in figure 1. The pipe length and diameter are selected as $6.4 \mathrm{~m}$ and $80 \mathrm{~mm}$ respectively, giving a lengthto-diameter ratio of $L / D=80$.

\subsection{Governing equations}

The governing equations, in cylindrical coordinates system, are as follows:

Continuity equation:

$$
\frac{\partial U}{\partial x}+\frac{\partial V}{\partial r}+\frac{V}{r}=0
$$

The momentum equation in the $x$ direction:

$$
U \frac{\partial U}{\partial x}+V \frac{\partial U}{\partial r}=-\frac{1}{\rho} \frac{\partial p}{\partial x}+v\left(\frac{\partial^{2} U}{\partial x^{2}}+\frac{1}{r} \frac{\partial U}{\partial r}+\frac{\partial^{2} U}{\partial r^{2}}\right)
$$

The momentum equation in the $r$ direction:

$$
U \frac{\partial V}{\partial x}+V \frac{\partial V}{\partial r}-\frac{W^{2}}{r}=-\frac{1}{\rho} \frac{\partial p}{\partial r}+v\left(\frac{\partial^{2} V}{\partial x^{2}}+\frac{1}{r} \frac{\partial V}{\partial r}-\frac{V}{r^{2}}+\frac{\partial^{2} V}{\partial r^{2}}\right)
$$


The momentum equation in the $\phi$ direction:

$$
U \frac{\partial W}{\partial x}+V \frac{\partial W}{\partial r}+\frac{V W}{r}=v\left(\frac{\partial^{2} W}{\partial x^{2}}+\frac{1}{r} \frac{\partial W}{\partial r}-\frac{W}{r^{2}}+\frac{\partial^{2} W}{\partial r^{2}}\right) .
$$

\subsection{Boundary conditions}

At inlet, a swirl component is superimposed on Poiseuille flow. The swirl is formed through a combination of forced vortex in the core and free vortex in the annulus. This is similar to the distributions that were experimentally realized in the previous studies (Bali 1998, Parchen \& Steenbergen 1998). Thus, the boundary conditions at pipe inlet $(x=0)$ are:

$$
\begin{aligned}
& U(0, r, \phi)=U_{\max }\left[1-\left(\frac{r}{R}\right)^{2}\right] . \\
& V(0, r, \phi)=0 . \\
& W(0, r, \phi)= \begin{cases}W_{\max } \frac{r}{r_{\text {trans }}}, & r<r_{\text {trans }} \\
W_{\max } \frac{r_{\text {trans }}}{r}\left[\frac{R-r}{R-r_{\text {trans }}}\right], & r \geq r_{\text {trans }} .\end{cases}
\end{aligned}
$$

In equation (7), $r_{\text {trans }}$ is the radial location at which transition from forced to free vortex takes place. The bracketed term in the free vortex velocity distribution is included to ensure that the velocity goes to zero on the pipe wall.

At exit $(x=L)$, a fully-developed flow condition was assumed. The no slip condition $(U=V=W=0)$ was imposed at the pipe wall.

\subsection{Swirl analysis}

A suitable measure of the swirl in pipe flow is the swirl number, defined as the ratio of the total flux of angular momentum to the axial momentum flux (Bali 1998, Parchen \& Steenbergen 1998). It is expressed as follows:

$$
S=\frac{2 \pi \int_{0}^{R} U(r W) r d r}{\pi R^{3} U_{a v}^{2}} .
$$

\section{Method of solution}

The dynamics of confined swirl flow is a complex one because of the co-existence of the axial and tangential components of velocities at any point of the flow field and the boundary layers at wall are three-dimensional. The flow is therefore not easily amenable to analytical solution, except in the core region where the flow can be considered to be invscid. Here, application of Euler's equation (Crowe et al 2005) shows favourable pressure gradient towards the vortex center. This results in the acceleration of the radial flow towards the center, which, in turn, leads to deceleration of the axial flow in order to satisfy continuity equation. As the strength of the swirl weakens downstream due to viscous dissipation, the original Poiseuille profile of the axial flow is gradually recovered.

The model equations 1-7 were solved numerically. Due to the axisymmetric flow situation, the computational domain becomes two-dimensional and the symmetry axis passes through 
the pipe center as shown in figure 1. A rectangular grid system was employed and the gridindependence test was conducted, which yielded a grid independent solution for $40 \times 600$ grid points.

Computation was made using the finite volume method. A staggered grid arrangement was used in order to prevent a 'checkerboard' pressure field (Patankar 1980, Versteeg \& Malalasekera 1995). The SIMPLE algorithm (Patankar 1980, Versteeg and Malalasekera 1995) was used to obtain the numerical solution.

Different inlet swirl numbers were realized by varying the values of $W_{\max }$ in equation (7). The effect of the nature of the inlet swirl distribution was investigated by varying the value of $r_{\text {trans. }}$.

\section{Results and discussions}

In the present study, the decay of swirl in laminar pipe flow was investigated numerically. The pipe dimensions were selected as $80 \mathrm{~mm}$ diameter, $6.4 \mathrm{~m}$ length. Computations were performed for six different Reynolds numbers $(\mathrm{Re}=$ of 800, 1000, 1200, 1400, 1600, 1800) and four inlet swirl numbers $\left(S_{o}=0.5,1.0,1.5,2.5\right)$, with four different inlet tangential velocity distributions $\left(r_{\text {trans }} / r_{o}=0 \cdot 5,0 \cdot 6,0 \cdot 75,0.9\right)$. In the results to be presented in figures $2-6$, the symbols are included for visual aid only; they do not represent the number of nodes used in the computations.

The axial velocity distributions in the pipe for swirl number $S_{o}=1 \cdot 0, R e=1000$ and $r_{\text {trans }} / r_{o}=0.75$ are presented in figure 2 , where it is revealed that the fully-developed (Poiseuille) velocity distribution at inlet is altered downstream due to the introduction of swirl. This is consistent with the observation made at the beginning of section 3 . The flow gradually recovers from the destabilizing effect of swirl (occasionally by adverse pressure gradient in the axial direction) and the initial profile is almost fully recovered at the pipe exit.

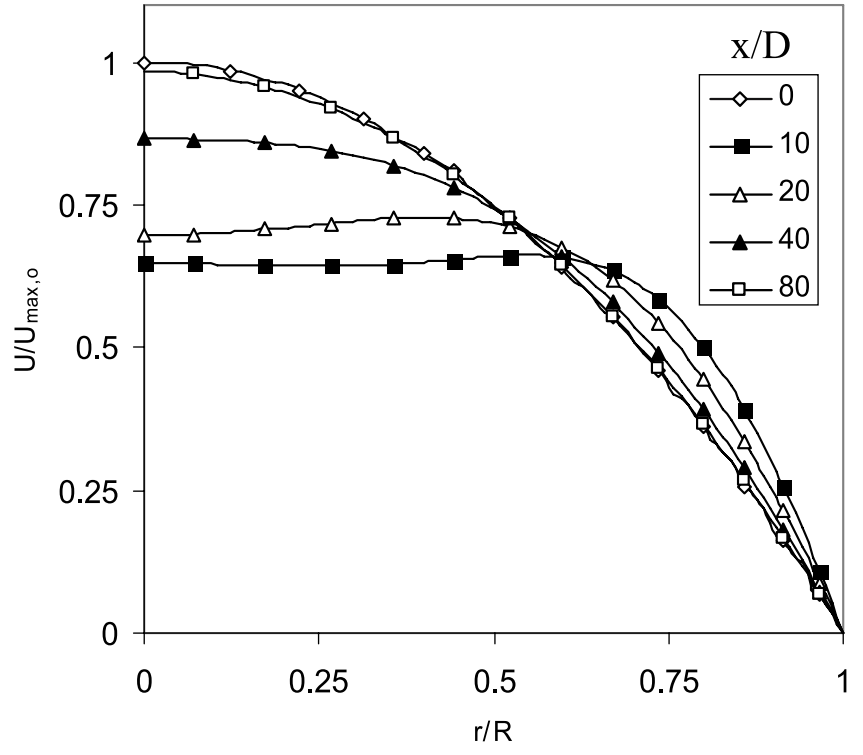

Figure 2. Axial velocity distributions in the pipe for $S_{o}=1 \cdot 0$, $\mathrm{Re}=1000$ and $r_{\text {trans }} / r_{o}=0.75$. 


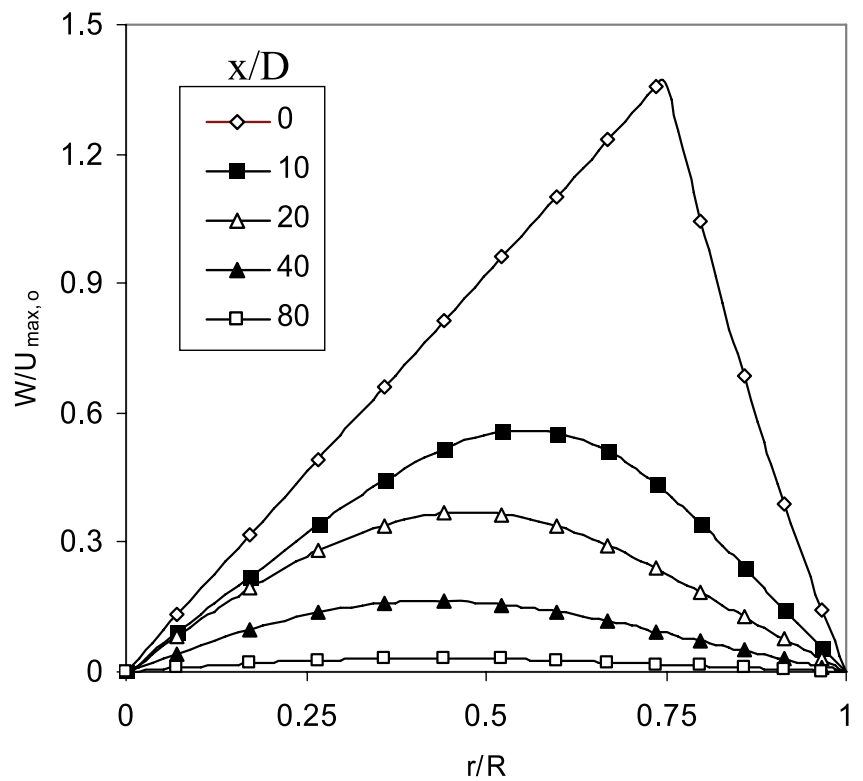

Figure 3. Tangential velocity distributions in the pipe for $S_{o}=1 \cdot 0$, $\operatorname{Re}=1000$ and $r_{\text {trans }} / r_{o}=0.75$.

Similar trends are obtained for other values of $S_{o}$, Re and $r_{\text {trans }} / r_{o}$ but are not shown here. The actual fully-developed profile will be recovered only if the swirl completely disappears and this requires an infinitely long pipe. A preliminary investigation revealed that the computed swirl decay, which is the subject of this study, is not affected by the finite length of the computational domain if $L / D>60$. The value of $L / D=80$ used in this study is therefore considered to be adequate.

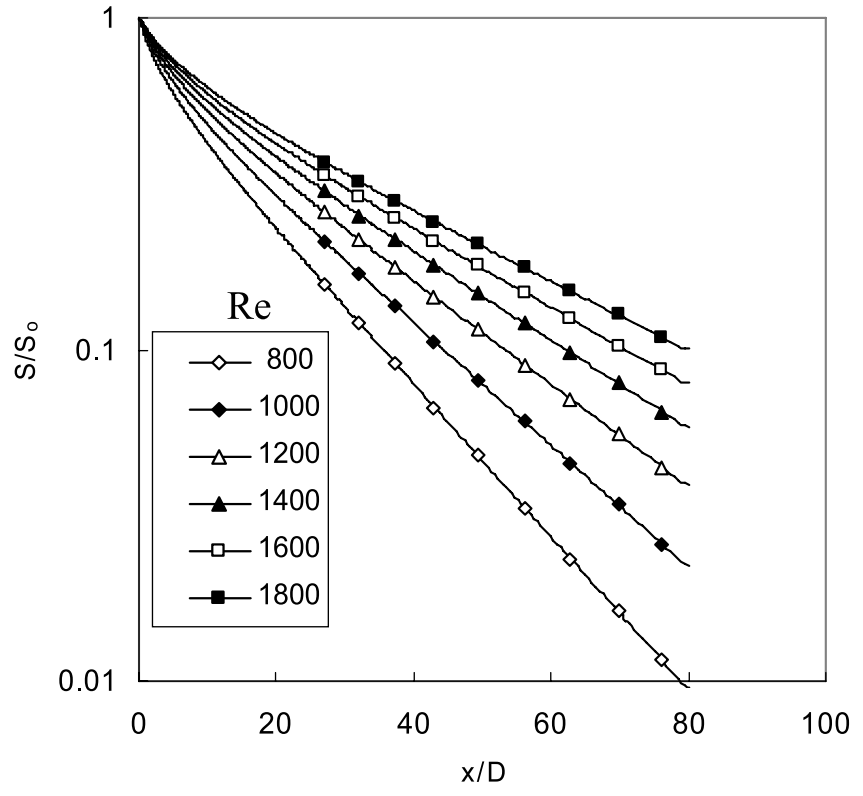

Figure 4. Decay of the swirl number along the pipe for $S_{o}=$ 1.0 and $r_{\text {trans }} / r_{o}=0.75$. 


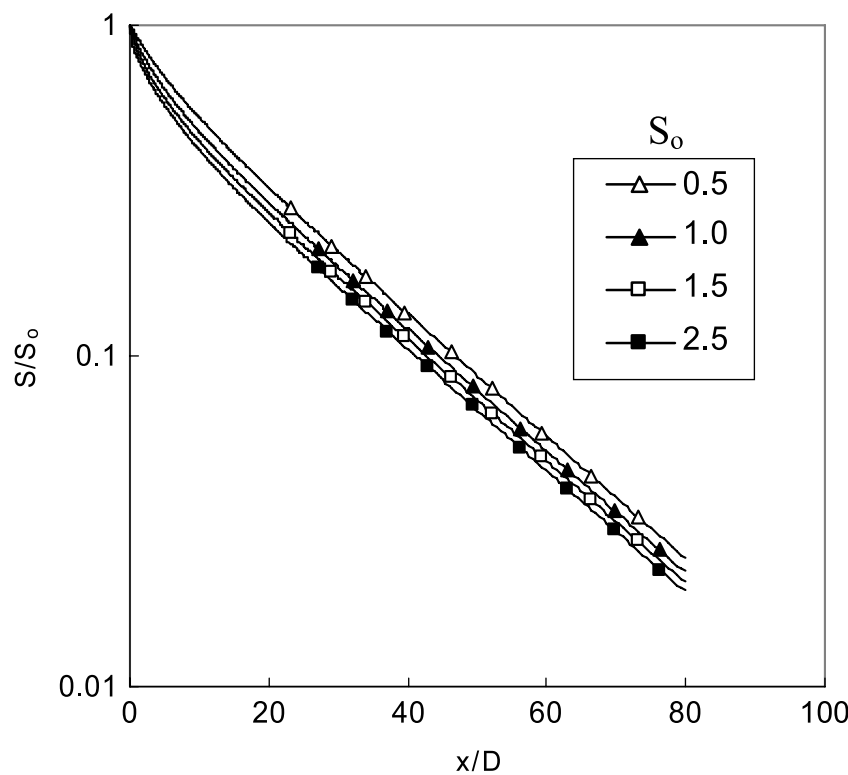

Figure 5. Decay of the swirl number along the pipe for $\mathrm{Re}=$ 1000 and $r_{\text {trans }} / r_{o}=0.75$.

Figure 3 shows the distributions of the tangential velocity in the pipe. The Figure reveals that as the flow progresses downstream, the swirl decays, the core region (for forced vortex) shrinks while the annular region (for free vortex) expands This trend was also reported by Chang \& Dhir (1995) and Bali (1998) for turbulent swirling flow.

The variation of the swirl number along the pipe length is shown in figure 4 for Reynolds numbers from 800 to 1800 and $S_{o}=1 \cdot 0$. It could be seen from the figure that after an initial

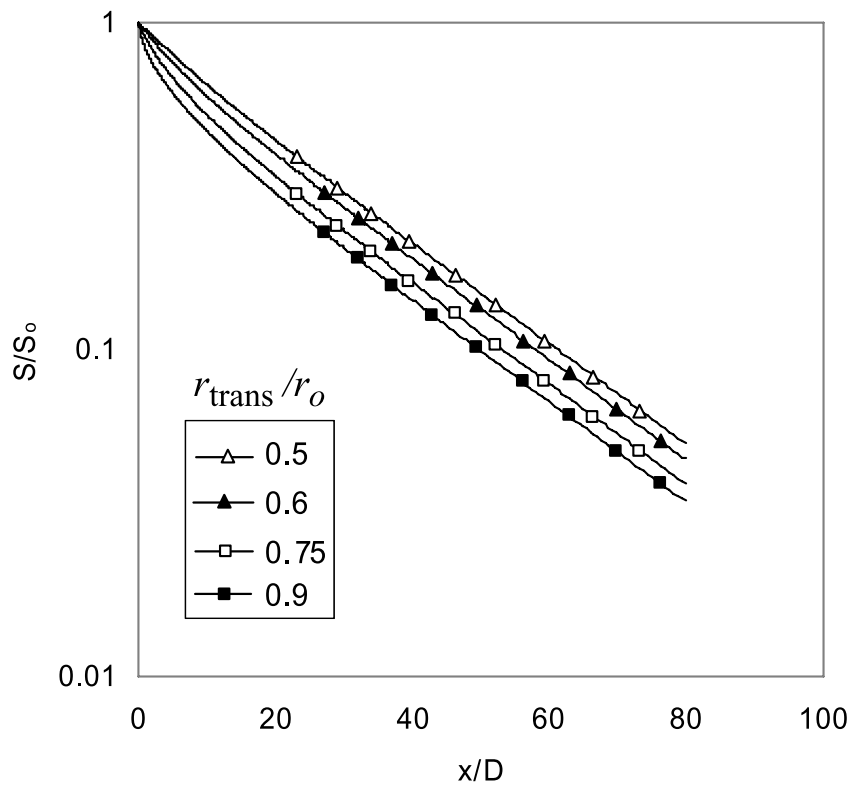

Figure 6. Decay of swirl along the pipe at $\operatorname{Re}=1200$ and $S_{o}=$ 1.0 for different distributions of inlet tangential velocity. 
rapid decay of the swirl, it then continues to decay exponentially towards the downstream. The figure also shows that the rate of decay decreases as the Reynolds number increases. The swirl decay at $\mathrm{Re}=1000$ for four levels of inlet swirl numbers are shown in figure 5 . The figure shows that the swirl number at pipe inlet does not affect the swirl decay rate, but its memory persists indefinitely. Figure 6 shows the decay of swirl along the pipe for different inlet tangential velocity distributions. Here, it is revealed that the swirl number at any downstream location depends on the nature of the inlet tangential velocity distribution. From figures 4 to 6 it is apparent that after the initial non-linear decay, the swirl decays linearly on a semi-log plot. This linear portion is found to be starting at $x / D=16$ in all the inlet conditions accommodated in the simulations. The swirl distribution can, therefore, be modelled in the form given below:

$$
\ln \left(S / S_{o}\right)=\ln C-m x / D .
$$

By using linear regression analysis (Montgomery 1997), the constants $C$ and $m$ were determined. It was founded that $\mathrm{m}$ is a function of Re only and its variation fits on a power function. $C$ varies with $S_{o}, \operatorname{Re}$ and $r_{\text {trans }} / r_{o}$. For each pair of $\operatorname{Re}, r_{\text {trans }} / r_{o}$, the dependence of $C$ on $S_{o}$ was investigated, and a power function was chosen as the best fit. The constants in the power function were modelled as combinations of linear functions of Re and $r_{\text {trans }} / r_{o}$. These were determined by linear regression. The equation for swirl decay is, therefore, presented as follows:

$$
S / S_{o}=C e^{-m x / D}
$$

where $m$ and $C$ are defined as

$$
\begin{aligned}
& m=25 \mathrm{Re}^{-0 \cdot 92} \\
& C=A S_{o}^{B} . \\
& A=7 \times 10^{-5} \mathrm{Re}-0.78\left(r_{\text {trans }} / r_{o}\right)+1 \cdot 2 . \\
& B=2 \times 10^{-5} \mathrm{Re}-0 \cdot 17 .
\end{aligned}
$$

Starting from $x / D=16$, the swirl distribution defined by equations (10)-(14) has been found to match the results of our numerical computation (for all $S_{o}$, Re and $r_{\text {trans }} / r_{o}$ ) to a maximum error of $1 \%$.

\section{Conclusions}

The swirl decay in laminar pipe flow with inlet swirl has been examined through a numerical computation of the flow field for four different inlet swirl numbers, six values of Reynolds number and four different tangential velocity distributions at pipe inlet. The swirl number distribution along the pipe was computed. A generalized relationship for swirl decay was then obtained by curve-fitting technique. The specific conclusions derived from the present study may be listed as follows:

(i) The introduction of swirl into a fully-developed laminar pipe flow distorts the usual parabolic velocity profile in the pipe. The profile is gradually recovered as swirl decays towards downstream. 
(ii) From the tangential velocity profile, the swirl flow can be divided into a core region and an annular region, characterized by forced-vortex and free-vortex types, respectively. As the flow progresses towards the downstream, the core region shrinks (reduces in size) while the annular region of free vortex expands.

(iii) The swirl number at any location in the downstream depends on the inlet swirl number, the flow Reynolds number, the distance from the pipe inlet, the pipe diameter and the nature of the inlet tangential velocity distribution. The swirl decays exponentially along the pipe length starting from $x / D=16$. The decay is correlated with a generalized relationship as defined by equations (10)-(14).

The support provided by the King Fahd University of Petroleum and Minerals in completing this work is acknowledged. The author is indebted to Prof. B S Yilbas for his encouragement and guidance throughout the work.

\section{List of symbols}

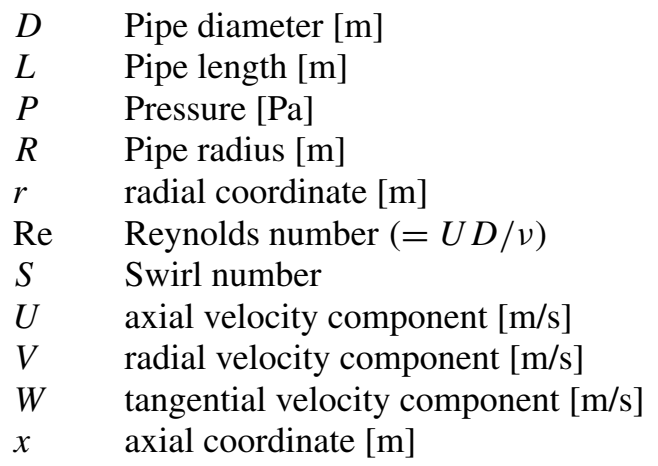

\section{Greek symbols}

$\phi \quad$ circumferential coordinate

$\rho \quad$ fluid density $\left[\mathrm{Kg} / \mathrm{m}^{3}\right]$

$v \quad$ kinematic viscosity $\left[\mathrm{m}^{2} / \mathrm{s}\right]$

\section{Subscripts}

$\begin{array}{ll}\text { av } & \text { average } \\ \max & \text { maximum } \\ \text { o } & \text { inlet } \\ \text { trans } & \text { transition point (from forced to free vortex) }\end{array}$

\section{References}

Bali T 1998 Modelling of heat transfer and fluid flow for decaying swirl flow in a circular pipe. Int. Comm. Heat Mass Transfer 25(3): 349-358 
Chang F, Dhir V K 1995 Mechanisms of heat transfer enhancement and slow decay of swirl in tubes using tangential injection. Int. J. Heat Fluid Flow 16(2): 78-87

Crowe C T, Elger D F, Robertson J A 2005 Engineering fluid mechanics. (USA: John Wiley), $8^{\text {th }}$ Ed. $113 \mathrm{P}$

Li H, Tomita Y 1994 Characteristics of swirling flow in a circular pipe. J. Fluids Eng. 116: 370-373

Montgomery D C 1997 Design and analysis of experiments (New York: John Wiley) $4^{\text {th }}$ Ed.

Parchen R R, Steenbergen W 1998 An experimental and numerical study of turbulent swirling pipe flows. J. Fluids Eng. 120: 54-61

Patankar S V 1980 Numerical heat transfer and fluid flow. McGraw-Hill, New York

Reader-Harris M S 1994 The decay of swirl in a pipe. Int. J. Heat Fluid Flow 15(3): 212-217

Versteeg H K, Malalasekera W 1995 An introduction to computational fluid dynamics: the finite volume method. (England: Longman)

Yapici S, Patrick M A, Wragg A A 1994 Hydrodynamic and mass transfer in decaying annular swirl flow. Int. Comm. Heat Mass Transfer 21: 41-51 